\title{
William Ritter entre la race et le sang : une exploration romanesque et ethnographique
}

\author{
Fabiana Fianco \\ Université de Lausanne
}

\begin{abstract}
Dans ses souvenirs, William Ritter affirme avoir toujours eu une « âme inquiète " et avoir aimé « tout ce qui n'était pas l'endroit présent, tout ce qui était ailleurs et au plus lointain ailleurs possible». Constamment attiré par « la passion de la planète » et par l'étranger, il a ainsi joué un rôle essentiel dans la découverte et la divulgation des cultures les plus ignorées et parfois les moins appréciées par ses contemporains. Le présent article explore le discours ethnographique de Ritter, qui révèle sa conception de la race, ses convictions antisémites et son rapport au sang et à sa symbolique. Il s'attache à montrer que la race est souvent pour Ritter un stéréotype assez figé, tant dans son écriture romanesque que dans ses rêves d'une édification nationale fondée sur le folklore.
\end{abstract}

Keywords: race, sang, slavité, antisémitisme, nationalisme

Voyageur inlassable depuis I889, William Ritter est considéré par Xavier Galmiche comme l'un des rares francophones fin-de-siècle à s'être défini comme un amateur des cultures de l'Europe centrale (Galmiche 2003). Dès sa jeunesse, il fait preuve d'une grande ouverture culturelle et il est assoiffé de connaissance, ce qui lui permet de développer une sensibilité unique envers les peuples les moins valorisés. Les voyages de Ritter se révèlent être ainsi la voie d'accès privilégiée à la découverte et à l'admiration de mondes jusque-là méconnus, que ses journaux et ses romans rendront présents. Néanmoins, le penchant ethnographique de l'auteur est également nourri par le contexte socio-culturel de la deuxième moitié du XIX siècle, particulièrement fécond en discours portant sur les concepts de race et de nation. Il est dès lors fondamental de replacer la pensée et les positions de Ritter dans le climat culturel de son époque, pour éviter d'en faire une lecture trop tranchée ou partiale.

Né en I867, Ritter est contemporain de la naissance et de la théorisation des premières études raciales au niveau international. En Europe, les nouvelles publications postulant l'inégalité naturelle des " races humaines" ont lieu en concomitance avec l'éclatement de la guerre de Sécession américaine (I86I-I865). Cette dernière est la conséquence tragique des débats sur fond éthique autour du système esclavagiste, débats qui affectent depuis longtemps aussi bien la politique américaine que l'économie colonialiste européenne. Frederick Douglass, héraut de l'abolitionnisme, identifie le premier le lien qui sous-tend les atrocités de l'esclavage : «l'orgueil et 
l'égoïsme, combinés avec les facultés intellectuelles, ne manquent jamais d'une théorie pour les justifier » (Taguieff 2003 : II). Son propos est symptomatique du délicat processus de rationalisation et de naturalisation du préjugé racial ayant cours au XIX ${ }^{\mathrm{e}}$ siècle, qui repose sur une série de théories données comme scientifiquement fondées.

Ce sont précisément ces théories qui expliquent le passage d'un racisme esclavagiste, qui avait habité la pensée européenne et américaine pendant la première moitié du siècle, et qui touchait avant tout les Noirs, à un racisme scientifique, dont la portée dépasse la question de la négritude. La typologie de rationalisation dominante est celle qu'on appelle "naturaliste ", dont les racines plongent dans la théorie darwinienne de l'évolution, réinterprétée dans une perspective sociale. C'est de ce ce mélange de discours que sont issues les doctrines politico-scientistes fondées sur l'idée de "race » (Taguieff 2003: 17) ; celles-ci présupposent une distinction essentielle et inédite entre le "racialisme ", entendu comme une élaboration idéologique à visée explicative, et le «racisme ", conçu comme un ensemble de normes et de valeurs conduisant à la discrimination et à la ségrégation.

Cette clarification est nécessaire lorsqu'on aborde le rapport entre Ritter et la race, car il recourt à l'une et à l'autre de ces notions, les adoptant en fonction du contexte qu'il aborde : la manière dont il se penche sur l'altérité slave relève du racialisme, alors que ses commentaires sur les Juifs révèlent un racisme ravageur. Sa vision est en phase avec son temps : comme le rappelle Taguieff, le racisme proprement dit est un phénomène relativement récent, qui connaît son apogée au cours du XIX siècle. C'est donc sur la base de la " construction de la catégorie classificatoire de la "race humaine" » (Taguieff 2003: 19) que Ritter parle des peuples avec lesquels il entre en contact, en se référant à des classements préétablis. Au moment où il commence à s'intéresser aux cultures étrangères, la notion de race est en effet idéologiquement et ethnologiquement configurée.

Une forme de proto-racisme existait cependant avant l'époque de Ritter ; elle était prioritairement liée à la notion de sang. Le passeur suisse semble parfois récupérer cette acception généalogique de la race, en vogue jusqu'au XVIII ${ }^{\mathrm{e}}$ siècle, pour la mêler à la science de l'homme de son temps. Sa représentation des liens du sang et de leurs implications déborde la définition d'un groupe familial pour inaugurer une vision positiviste et matérialiste selon laquelle les caractères phénotypiques sont l'indice de l'appartenance à une certaine nature de sang. La croyance en une telle appartenance a de multiples retombées : fondée sur l'affirmation du partage de qualités héréditaires supérieures ou inférieures, elle justifie la lutte pour la préservation de la pureté du sang, en réaction au déclin culturel et à la décadence biologique que causerait le mélange entre les peuples. 


\section{Un penchant slavophile}

Le remède que Ritter envisage contre la décadence raciale est incarné principalement par le modèle slave, qu'il vante d'autant plus qu'il est persuadé d'être investi d'une "mission de défense des peuples slaves et de la découverte de l'Europe centrale et des Balkans au profit des occidentaux » (Listīkovā 2008 : 6). Fillette slovaque (1903) apparaît ainsi comme un hommage à la pureté d'âme et de sang que les Slaves ont su préserver de la corruption. Nous allons analyser les passages du roman relatifs à la race et au sang en nous servant des instruments théoriques du racialisme gobinien et $\mathrm{du}$ racialisme biologique et nationaliste de Drumont et Soury'. Précisons tout d'abord que, par ce récit, Ritter postule l'existence d'une race slave qu'il envisage sous l'angle de la théorie gobinienne de "la race perdue ", selon laquelle "la disparition du "sang pur" par l'effet des mélanges interraciaux » conduit à la dégénéréscence raciale (Taguieff 2003: 25). Mais Ritter parle aussi de la race slave en termes eugéniques, dans la mesure où il la présente comme le produit d'une fabrication et d'une sélection volontaires, obéissant $\mathrm{au}$ " principe du déterminisme biologico-racial "telle race/telle culture" ou "telle race/tel ensemble d'aptitudes" »(29).

En effet, Ritter bâtit un imaginaire culturel, national et spirituel slave ad hoc d'après ce qu'il a vu pendant ses voyages et grâce à Janko Cádra, son deuxième compagnon slovaque. La plongée dans cet univers romanesque appelle dès lors une prémisse. Au tournant du siècle, Georges Vacher de Lapouge, anthropologue et théoricien de l'eugénisme, affirme que « toute "société" ou toute "nation" se compose d'individus appartenant à des races diverses (et inégales). Il n'y a donc ni de "race française", ni "race germanique", ni "race slave", ni "race israélite" (ou juive)»(243). La race serait ainsi une invention, voire une construction : d'où l'impossibilité de la définir selon l'appartenance géographique d'un peuple affilié à une seule « catégorie raciale ». Par conséquent, Lapouge conclut que la race est une réalité à créer, du moment qu'elle n'est plus assimilable à un bien naturel qu'il s'agirait de préserver de la décadence ou de la perte (29). Il nous reste à voir si et comment William Ritter a créé la race slave, et quel est le portrait qu'il en dresse.

Dans la dédicace à Janko Cádra, Ritter définit Fillette slovaque comme un " précieux coffret de travail paysan », la « révélation » d'un peuple, bref « un témoignage définitif de [son] estime, de [son] respect et de [son] amitié pour [Janko] » (Ritter 1903: 9), qu'il assimile à un parfait représentant de la race slave $^{2}$. Le projet de Ritter est clair : il veut faire de Cádra et de tout ce qu'il

I Pierre-André Taguieff identifie quatre figures de racialisme, aussi définies comme les quatre "types" idéaux : le racialisme gobinien/pessimiste ; le racialisme évolutionniste et darwiniste; le racialisme déterministe/antisémite ; le racialisme eugénique.

2 «Tu la ressembles toute et tu la magnifies » (7); « toi qui me sembles la patrie slovaque » (8); 
symbolise « un Slovaque enfin DONT NUL N'AIT LE DROIT D'IGNORER QU'IL SOIT UN HOMME »(9). Il œuvre donc au rétablissement de la culture slovaque et de la slavité qu'elle implique; plutôt que de « race slovaque », Ritter préfère en effet parler de « race slave ». Les très nombreuses récurrences du terme « slave » dans le texte ont été étudiées par Cécile Gauthier, qui observe « un intérêt marqué pour ce nom et ses implications » (Gauthier 2008 : 2) ; sa valeur souvent explicative permet selon elle « de comprendre le geste créateur d'un artiste d'Europe centrale, ou encore, au sein de la fiction, de motiver l'action d'un personnage, et ce d'autant plus que cette action étonne et paraît contraire à la logique » (2).

Aussi, le comportement inexplicable de Martin qui, follement amoureux d'Anička, accomplit un geste de vénération, est réinterprété à la lumière de la race slovaque : "C'était si peu, si peu slovaque ce qu'il allait demander là... D'où avait bien pu lui germer cette idée, de quelles autres lointaines hérédités slaves... »? (Ritter I903 : 270). Quand il parle de slavité ou de race slave, Ritter ne se réfère pas seulement aux Slovaques, mais également aux Russes et aux Tchèques, bien que les Russes, comme le précise Gauthier, fassent plutôt partie d'une slavité orientale (Gauthier 2008 : 9). La race slave occidentale, quant à elle, est associée principalement à la paysannerie et à la rusticité ; les Slovaques de Ritter sont des âmes pures et sauvages, gardiens de l'authenticité de la vraie slavité, attachés à un patrimoine ancestral de traditions et de coutumes qu'ils cherchent à protéger et à conserver ${ }^{3}$, puisqu'ils ont eu « tant à feindre pour un peu sauvegarder leur vie nationale si compliquée » (Ritter I903: II2). Leur simplicité les rend presque naïfs, d'où leur spontanéité innée et leur penchant naturel pour les émotions plutôt que pour la raison. Ritter met également l'accent sur la solidarité mutuelle qui existe entre les Slaves « de partout », unis par la même "symphonie nationale » : ces « diverses patries » sont loyales envers une même race (29I-292). Cette dernière déborde ainsi les frontières politiques, car c'est le partage d'une nature slave commune qui signe l'appartenance à un même réseau de références culturelles.

Cette vision idyllique a de quoi éveiller le soupçon ; Cécile Gauthier confirme la légitimité des doutes suscités par cette image que dicte une slavophilie idéale, ne fût-ce qu'en rappelant, à travers une étude lexicogra-

« la fenaison entière de tout ce que j'ai aimé de ta nation avant toi, de tout ce dont tu incarnes et transmues et vivifies la vertu, de tout ce à quoi tu ajoutes une persuasion de plus » (IO).

3 Anička est le moyen par lequel Ritter montre le désir de conservation des coutumes slaves traditionnels : « Pour ces courses, Anička mettait ses meilleures bottines. Depuis longtemps, hélas ! les Slovaques de Neudorf ont abandonné l'opanka traditionnelle slave [...] qui est un signe distinctif de la race » (56); «Une chemise de son pays » et « le bonnet des filles de son pays » (58). Et quand elle voit Juro après longtemps, elle remarque qu'il « [porte] avec une rare élégance et une grâce suprême le vrai, l'intégral costume de sa race, l'un des plus typiques même» $(83)$. 
phique, la diffusion massive du mot « slave » dans les dictionnaires de la première moitié du XIX ${ }^{e}$ siècle, période qui coïncide avec les revendications de défense de l'identité slave. Cette dernière a fait l'objet d'un long processus de construction, cimenté par une tendance à l'idéalisation et par la création d'un imaginaire ${ }^{4}$. Ritter s'inscrit dans cette vague, même s'il le fait de façon paradoxale, en faisant coïncider réalisme et stéréotype national. Ce dernier n'affecte pas seulement son écriture romanesque, mais oriente aussi sa vision de l'altérité lorsqu'il est en voyage.

Dans Fillette slovaque, le stéréotype par excellence de la slavité est l'Exposition nationale tchécoslovaque de Prague de I895. Ritter l'envisage comme « une éclatante affirmation » de la pureté slave et d'un esprit national depuis longtemps réprimé par la persécution d'une "glorieuse pauvre race " (25I). L'exposition a pour but de "glorifier [les Slovaques] mieux que toute revendication politique », en se dressant contre toute menace de dénationalisation ou imposture morale (87). Cette aspiration est portée par Anička, l'héroïne du récit, appelée à lutter pour un « très idéal » petit bien-aimé.

\section{Anička, ou l'incarnation de la slavité}

Anička est le cœur de l'univers slave idéalisé par Ritter, qu'elle incarne physiquement : elle est "très blonde ", aux " cheveux d'or cendré [...] plus pâle que sa peau », elle a des "yeux très clairs, les yeux de sa race, bleus ou gris ou verts », et une « taille de long insecte serrée dans le corsage » (Ritter 1903: 56). Selon Gauthier, « le stéréotype du personnage slave se caractérise par sa blondeur délicate, sa grâce et sa fragilité » (Gauthier 2008:8) : Ritter reproduit donc une physionomie et une corporalité figées, l'azur des yeux revenant par ailleurs comme un trait récurrent. Anička est une "primitive petite paysanne slovaque ", "sage de nature " et avec une " âme naïve "; elle est " candide et ignorante", dotée d'une "simplicité rustique", et son attitude est « charmante de grâce sans apprêt » (Ritter 1903:67). Le personnage est tellement épuré, qu'elle craint elle-même de tomber dans ce qu'elle appelle la «perversion de la personnalité populaire» (6I).

L'évolution d'Anička au long du récit est tout intérieure : son parcours l'amène à prendre conscience de la race slave qu'elle symbolise et qui est la sienne. Contrairement à la vision d'un Gobineau, selon lequel il existe des races plus ou moins perfectibles, c'est-à-dire plus ou moins à même de se rapprocher du modèle de perfection qu'est la race aryenne, Ritter n'inscrit pas la slavité dans une dymanique de perfectibilité : Anička demeure tout au long du roman identique à elle-même, car sa nature sauvage, pure

4 Gauthier affirme que « le nom slave serait davantage source de rêveries qu'un nom de nationalité au sémantisme souvent plus circonscrit et moins indéterminé » (2008: 2). 
et paysanne est accomplie en soi et ne doit pas être altérée. La doctrine générale de l'époque au sujet des races privilégie l'évolution de la sauvagerie à la civilisation ; dans sa vision de la slavité, Ritter semble plutôt proche des mouvements primitivistes qui émergent à la fin du XIX ${ }^{\mathrm{e}}$ siècle, pour lesquels la sauvagerie est à la fois un abri protecteur et une garantie d'authenticité. De ce fait, la conception de la race qu'il envisage semble anticiper ce qu'on appellera plus tard le "néo-racisme », basé sur le paradigme du relativisme culturel et prônant " la séparation entre les groupes humains différents comme la seule politique réaliste » (Taguieff $2003: 33$ ).

Le métissage, voire le mélange des races, comment Ritter les aborde-t-il ? Sa conception de la slavité ne va pas de soi, dans la mesure où elle englobe plusieurs nationalités, notamment les Slovaques et les Tchèques. Dans Fillette slovaque, les deux nationalités apparaissent comme équivalentes, parce qu'elles partagent une même pureté d'âme et une culture enracinée dans la paysannerie ; elles sont en fin de compte deux branches d'une grande famille. À l'échelle européenne, en revanche, Ritter n'encourage pas le métissage, qu'il envisage plutôt comme une source potentielle de décadence et de contamination irréversibles ; il rejoint par là Gobineau, qui affirme que « les peuples ne dégénèrent que par suite et en proportion des mélanges qu'ils subissent » (Taguieff 2008 : 5I). S'il relativise dans certains cas l'idée de l'incompatibilité naturelle des races, Ritter n'est favorable à la rencontre des cultures que dans un cadre et des conditions bien précis.

Ainsi, trois menaces pèsent sur la pureté d'Anička : le Juif(Rudi) ; la civilisation (Vienne) ; l'étranger (Juro et avec lui la Hongrie). Du dernier de ces éléments perturbateurs, Juro, Ritter dit qu'il n'est « point un Slovaque pursang » (Ritter I903: 56), et son altérité par rapport à la nature d'Anička est mise en évidence : "En elle résidaient toutes les vertus humbles et tendres, toutes les menues gentillesses et la gracilité timide opposées à sa crânerie de beau mâle irrésistible, bronzé, noiraud, aux yeux fauves, comme si, en lui, le Slovaque, par quelque hasard fréquent sur ces frontières, avait été mâtiné de Hongrois » (36). La différence entre leurs deux essences éclate quand Anička se rend à Prague et découvre le « chef-d'œuvre » de la race slave, représenté sur l'affiche de l'Exposition nationale d'Hynaïs. Anička tombe littéralement amoureuse de l'image : l'artiste tchèque a réussi à créer un « véritable type " slave, « la synthèse des jeunes hommes slaves de la campagne " (9I) qu'elle idéalise et qu'elle appelle Janko. Le choix qu'elle a à faire entre les deux garçons est plus simple dès lors que Juro devient une source de trouble et de corruption, après avoir subi une sorte de métamorphose qui lui fait quitter l'idéal paysan et le dépouille de son authenticité : «Oh ! Comme il ressemblait peu à l'autre Juro, décidément le vrai, celui-là, celui de l'affiche de Prague» (IO4), « celui qui était le vrai pour elle» (I43). 
Quand Anička découvre que le modèle de l'affiche existe, elle veut le rencontrer à tout prix, subjuguée qu'elle est par son image. La description que Martin fait de Janko coïncide avec le portrait d'Anička : doté d'une « divine simplesse paysanne » et d'une « virginité amie », il évolue dans un cadre bucolique de «vie primitive» (307). À plusieurs reprises, Ritter souligne ce dernier aspect chez le personnage, qui, sous un " toit primitif », mène " la vie primitive d'autrefois " (309). À peu de chose près, Janko est une réactualisation du bon sauvage, dont la caractérisation est compatible avec le racialisme de Gustave Le Bon, pour qui la sauvagerie et la pureté de la race dérivant du sang vont de pair. Dans cette optique, le sauvage incarnerait la race la plus pure, puisque préservée de toute contamination civilisatrice. Martin dit explicitement ne pas vouloir troubler la "sauvagerie de Janko " (308), qui n'abandonnerait pour rien au monde les forêts de sa montagne pour le « pays de bouleaux » civilisé (3I0).

La relation entre les deux univers antinomiques est matérialisée par l'image d'Anička aux pieds nus : «Il me semble que je ne pourrais jamais aimer une fille slave autrement que pieds nus ou chaussée d'opanky... Les souliers c'est bon pour être servante chez les Nemči » (313). Le détour par Prague déclenche chez Anička la réappropriation de sa slavité, dans le rejet du travail de domestique qui symbolisait la corruption du civilisé : «[Elle] avait appris à prendre conscience de sa race. Elle était devenue femme et presque demoiselle et même aussi mieux Slovaque à Vienne; elle devint de Slovaque, Slave, à Prague » (227). Grâce à Janko et à la prise de conscience qu'il provoque, Anička peut être avec lui la garante d'un nid sauvage imaginaire, sans taches et isolé du monde, capable de sauvegarder l'essence slave et de la protéger de toute atteinte, y compris de l'atteinte juive.

\section{Ritter antisémite?}

La plupart des études consacrées à Ritter rappellent l'antisémitisme qui émaille sa production romanesque et les impressions tirées de ses nombreux voyages. Politiquement d'extrême droite et irrité par les bourgeois, Ritter adhère pendant sa jeunesse à la cause antisémite, dont l'affaire Dreyfus montrera peu d'années après la virulence. Il soutient dans ce sens la violente politique antisémite menée par le maire Karl Lüger à Vienne, où il déménage en I888. "Son antisémitisme y trouvera un aliment régulier » (Donzé 1999 : 87), à un moment où la vulgate gobiniste est mise au service d'un germanisme fondé sur la régénération raciale des lignées aristocratiques, contre la contamination par le sang « vil et abject " (Taguieff 2008: 20). Ce « sang » est notamment attribué à un ennemi que toute race noble se doit de combattre pour maintenir son unicité : le Juif. 
Au cours d'un processus de démonisation intense pendant la deuxième moitié du XIX ${ }^{\mathrm{e}}$ siècle, le Juif devient une sorte de «type » racial dont il faut se méfier, d'une part à cause de la « nature » qui lui est prêtée, d'autre part en raison de la suspicion dont il fait l'objet dans le domaine politique et financier. En cette période où le nationalisme triomphe, la théorie des races trouve dans le Juif le contre-exemple à proscrire, et la xénophobie généralisée est programmée pour se transformer en judéophobie, dans la mouvance de ce qu'on appelle le national-racisme. Ainsi Ritter ne prévoit-il une régénération raciale que pour les peuples «celto-slavo-germaniques » (73), les Juifs en étant d'office exclus. Il est intéressant de savoir que Fillette slovaque était à l'origine prévu comme le premier volet d'une tétralogie dont la dernière partie aurait dû s'intituler « Judapest » (Szabó 2008 : 5) ; le texte apparaît ainsi comme emblématique du rapport de Ritter au Juif.

Quand Anička déménage à Vienne au service de Madame Wieland, on nous apprend que celle-ci a adopté un " petit misérable ", Rudi, abandonné devant sa porte, « leur infligeant la plus cuisante des mésaventures » (Ritter I903 : 64). Ritter le décrit comme "un petit être » aux cheveux et aux yeux noirs et au teint pâle, « un bel et bon petit juif » au milieu « des autres tout ronds, blonds, roses, camards »; l'auteur insiste fréquemment sur cette différence physique $(65,68,69)$. Ce portrait est à lui seul symptomatique du climat de haine raciale propre à l'Europe de l'époque : si l'image de Rudi est aussi détaillée et complète, c'est que Ritter véhicule un imaginaire selon lequel le Juif est reconnaissable à partir d'une série de traits somatiques, psychiques et culturels. Toutefois, le Juif est également dangereux à cause de ses capacités de camouflage et d'imitation des Aryens ; chez Rudi, la dissimulation est rendue possible par l'éducation catholique et autrichienne qu'il a reçue. D'où une mise en garde capitale : « un bon antisémite doit savoir et pouvoir voir les Juifs » (Taguieff 2003 : I45). N'est-ce pas là ce que fait Ritter ? Son effort tend précisément à assimiler Rudi au Juif, en dépit du fait qu'il a été élevé dans un milieu catholique parfaitement aryen5. Rudi a beau se camoufler, un trait distinctif le trahit toujours, son « glorieux nez israélite » (Ritter I903 : II8) ; c'est pourquoi, après qu'il a été agressé par Juro, Madame Wieland voit dans le fait qu'il a désormais le nez cassé un avantage quasi providentiel : « elle se calma un peu à l'espérance que sans doute on ne s'apercevrait plus que Rudi fût juif » (I2O). Le contraste physique ne s'estompe pourtant pas : Ritter insiste sur le physique aryen des frères autrichiens de Rudi et d'Anička, de Martin et de Janko. Rudi devient ainsi sous sa plume l'emblème de l'éternel errant et de celui qui, étranger partout, salit

5 Ægyptiaque offre un autre témoignage de camouflage juif démasqué en référence à Neuenhaven : "Sans que nul s'en fût jamais douté, de cette race de Juifs blonds, d'autant plus heureux en affaires qu'ils parviennent souvent à se départir de tous les traits physiques de leur race » (Ritter I89I : I66). 
et corrompt : on ne se défait pas de son hérédité raciale, et si l'on tient au salut de ses descendants et au respect des ancêtres, le Juif est à proscrire. Dès la première rencontre, Anička identifie et rejette instinctivement Rudi : «Avant de l'avoir vu, elle avait reconnu Rudi, le jeune juif », et d'emblée elle lui « témoign[e] sa subite haine et son instinctive répugnance de toujours » (69-70). Systématiquement placé en position d'infériorité par rapport à la race slave incarnée par Juro, «beau garçon brun et fort de son pays » (72), Rudi est perçu négativement à travers les yeux de la jeune fille : « hypocrite et cachottier, paillard et salace jusqu'à l'immonde ", cet " être à la nauséeuse pâleur » est « lâche à la juive », et se caractérise surtout par sa « perfidie » et par « une féroce hypocrisie » (66-74).

La méfiance d'Anička s'étend du reste aux Juifs dans leur ensemble; de par leur présence, Vienne apparaît abîmée par « le hideux bariolage américano-juif » (8o), et Ritter insiste sur la dégradation de l'espace public dont ils seraient la cause et la manifestation : «C'était plein de hideux voyous variolés, vérolés, tous dans des défroques de gigerls usées, vingt fois retapées et dont l'élégance d'antan devenait du vice sur leurs corps » (I45). Sans surprise, on s'attend dans le roman à ce que le Juif soit mêlé à des affaires déloyales; ainsi Anička craint-elle « un indice qui pût lui suggérer quelque mauvais coup de juif, c'est-à-dire quelque gain à sophistiquer, quelques florins à s'approprier malhonnêtement » (I45). L'antisémitisme rittérien assume ainsi une dimension socio-culturelle, ce qui révèle une intolérance vis-à-vis de la communauté juive dépassant le strict univers fictionnel.

\section{Antisémitisme hors du romanesque}

L'antisémitisme du roman de Ritter interpelle : ce tableau judéophobe fait-il partie de la fiction ou est-il du ressort de l'auteur lui-même ? À la lecture d'autres écrits de Ritter, sa haine raciale pour les Juifs ne fait pas de doute. Contrairement à ce qu'on a pu penser, son antisémitisme n'a pas été nourri par son admiration pour Wagner, comme le rappelle Caroline Calame : «On aurait tort [...] d'imputer à ce dernier l'antisémitisme de Ritter, antipathie qui lui fait attribuer à des Juifs la plupart des rôles désagréables. [...] Cette intolérance était partagée par de nombreux contemporains et il n'est que de lire Proust pour mesurer l'antisémitisme qui caractérisait alors la société française» (Donzé I999: 79).

L'antisémitisme de Ritter n'est d'ailleurs pas sans contradiction, comme le révèle la relation à Gustav Mahler qui, quoique juif, devient une de ses références musicales majeures (Fournier-Facio 2010 : 32I). Cette élection ne modifie cependant pas sa position fondamentalement hostile à toute influence hébraïque, ce qui est du reste confirmé par les propos de Cádra (8). Mahler est une exception, et son judaïsme est en quelque sorte racheté par 
les liens qui le rattachent à l'univers slave : " [...] con Mahler, si tratta di un'altra Austria, da un lato con un fondo piuttosto slavo nello stato d'animo e, dall'altro, piuttosto alpestre nei paesaggi : la Terza Sinfonia, [...], evoca ben più le campagne della Boemia e richiama le impressioni dellinfanzia a Kalischt » (Fournier-Facio 2010 : 318) ${ }^{6}$. Malgré son idéal de pureté slave, Ritter accepte avec Mahler l'« immixtion des identités nationales et raciales », et le mélange d'éléments slaves et germaniques (Gauthier 2008 : II). À ses yeux, Mahler est « le plus Autrichien des musiciens contemporains, surtout par ceci qu'il est slave, beaucoup plus slave qu'allemand » (Meylan 2000 : 319-320). Selon Gauthier, Ritter désigne par « Autrichien » non seulement la composante germanique, mais une vision supranationale qui débouche sur ce qu'elle appelle l'« austrosalisme » (Gauthier 2008 : II) ; être Autrichien signifierait donc être slave « verni de germanisme » (Meylan 2000 : 319). Dans cette perspective, Ritter peut réinterpréter positivement l'hébraïsme mahlerien, et admettre « derrière lui la poussée irrésistible de toute la puissance juive d'alors » (Donzé I999: 46).

Les voyages de Ritter à travers l'Europe révèlent un antisémitisme généralisé. En Roumanie, les Juifs sont des « désagréables comparses » (Ritter I894: 7) qui forment une "dynastie nouvelle et étrangère » réduisant les motifs nationaux à un « souvenir historique» (Ritter I895 : 98). En Slovaquie, la famille de Cádra alimente le racisme de Ritter, d'autant plus que le jeune homme est né au moment où l'antisémitisme devient partie intégrante du nationalisme slovaque (Szabó 2008 : 2). Dans les notes et les cartes postales du voyage qu'il a effectué en Hongrie avec Ritter, Cádra signale à plusieurs reprises le mécontentement causé à son compagnon par les logements que gèrent des Juifs (3-4). Toutefois, les deux hommes ne sont pas forcément d'accord sur la question ; Cádra ne partageait pas toujours l'antisémitisme de Ritter, et il critiquait "the "shrillness" and vulgarity of anti-Semitic newspapers [...] that belonged to Ritter's reading. Sometimes Cádra objected to Ritter's literary work for the same reasons » (4). Pour sa part, Ritter voyait l'antisémitisme de Cádra comme une manifestation typique du nationalisme de son pays, selon lequel les Juifs menacent le folklore et l'authenticité slovaques ; lui-même, Ritter, tend en revanche à universaliser le rejet qu'il exprime. L'apport de Janko à l'antisémitisme de Ritter est plus intéressant si on l'aborde dans la perspective de Xavier Galmiche, qui propose de l'interpréter « comme le symptôme d'une transposition d'une altérité insupportable, depuis une instance sexuelle sur une figure sociale» (Galmiche 2008 : 2I). Après avoir mis au jour les liens entre la vie intime de Ritter et la représentation de la

6 « Avec Mahler, on a affaire à une autre Autriche, avec d'un côté un fond plutôt slave lorsqu'on considère son état d'âme, et, d'un autre, plutôt alpestre lorsqu'on se penche sur ses payages : la Troisième Symphonie [...] évoque davantage la campagne de Bohême, et rappelle les impressions de l'enfance vécue à Kalischt ». 
sensualité et de la sexualité qu'il donne dans sa production romanesque, Galmiche se concentre ensuite sur le corps des Juifs :

Pour cet observateur obsédé de sa marginalité, le Juif, ou plutôt le corps des Juifs, est sans doute le marginal subsidiaire sur lequel transférer la hantise de la souillure dont il s'est lui-même lavé. [...] [J]'interprète la relation à l'homosexualité assumée et à la judéité abhorrée comme le moment de la «crise mimétique », où le rôle traditionnel du Juif comme bouc émissaire est réactivé à nouveaux frais. Le corps des Juifs, dont le spectacle provoque une réaction immédiate de rejet, est le tabou absolu qui a permis la dilution des tabous intermédiaires (2I).

Galmiche ne se borne ainsi pas à expliquer l'antisémitisme de Ritter en le situant dans le contexte raciste de l'époque : en le rattachant à l'homosexualité de Ritter, il voit dans l'aversion pour les Juifs une haine d'abord liée à sa sexualité. La vague homophobe nazie, dont Ritter verra les effets destructeurs, mettra du reste sur le même plan Juifs et homosexuels, en stigmatisant la «saleté » de leur sang.

\section{Symbolique du sang}

Ritter aborde la question du sang dans une double perspective. D'un côté, il la thématise dans son discours portant sur la race; de l'autre, il traite le sang comme un élément en quelque sorte autonome, ayant une portée symbolique distincte.

Lorsque la pensée gobiniste de l'époque envisage le sang en tant que fondement de la race, elle établit des échelles de valeur et des hiérarchies : d'où le prestige du «sang bleu ». La noblesse porterait ainsi en elle un ensemble de qualités propres au sang d'un peuple, dont elle serait l'émanation la plus parfaite, à protéger de la corruption que tout mélange interracial lui ferait subir (Taguieff $2008: 46$ ). Certains tenants du déterminisme biologique et certains amateurs des théories de l'hérédité naturalistes rêvent même d'une communauté au sein de laquelle chacun aurait sa place selon la qualité de son sang. Ces visions sont bien sûr dépourvues de tout fondement scientifique et reposent sur des constructions imaginaires : « le lien du sang n'est pas une notion biologique, mais une fiction sociale» (Kozakaï 2006 : 193). C'est bien cet univers fantasmé que reproduisent les romans de Ritter, notamment quand ils peignent la slavité et la judéité.

Sa production romanesque témoigne par ailleurs une attention particulière à la généalogie des personnages. Le sang y est traité comme un élément porteur d'un déterminisme individuel, qui influence tant les actions que les pensées des sujets en présence. Egyptiaque et Âmes blanches sont les textes 
qui insistent le plus sur le sang en tant qu'indice de noblesse de race. Ainsi, en dépit de son histoire familiale peu orthodoxe, Ægyptiaque, par son sang, est de plein droit une Wesselitchko : «Les Wesselitchko sont mêlés, sous tous les rois de Pologne, à la grande histoire de leur pays. Écrire leur généalogie serait récrire les annales de leur patrie ; toucher à eux, c'est toucher à la Pologne héroïque et légendaire. [...] Partout où fut versé le sang de la Pologne coula du sang des Wesselitchko ( Ritter I89I : 39). Parmi les nobles, la jeune femme apparaît comme une étrange exception, étant vue comme une orpheline ; mais sa noblesse de sang reste indiscutée. Sa qualité d'aristocrate est un des enjeux de ses relations amoureuses. Hermann Thor, le pianiste de talent éperdument amoureux d'elle, craint qu'une jeune princesse ne puisse que rejeter un artiste pauvre, et essaie de déjouer les impératifs du sang. Ægyptiaque, elle, se dit prête à s'attacher à quiconque possède un certain goût de noblesse (4I), et elle table sur une manière d'équivalence entre son « aristocratie de race » et « l'aristocratie de génie » de Thor (8I). Elle peut ainsi faire fi des lois dictées par la croyance à la pureté de sang, et admettre Thor et Neuenhaven à ses côtés, en affirmant qu'« une princesse polonaise est capable de tout en présence d'un prince selon le génie » (I59). On devine, derrière ces propos, l'image que Ritter se fait des artistes et des génies créateurs, qui par leur aristocratie d'âme et d'esprit deviennent des élus placés sur le même plan que les nobles de naissance.

Cette thématique sous-tend aussi Âmes blanches, où le lien entre sang et noblesse est omniprésent. Théodule de Praroman, jeune aristocrate fribourgeois, y est décrit comme un «petit noblot, entiché de sa race » (Ritter I893: I4), au " nom célèbre dans les annales de la Suisse aristocratique et patricienne » (7). Sa famille vante une généalogie « s'arrêtant aux héros les mieux marqués » (57) et remontant à « la branche de Savoie, l'unique glorieuse et la plus noble » (55). Ritter insiste sur le « sang absurdement pur » et «bleu » (63) de son héros, qu'il oppose à celui, «trop épais », de Raoul (I80). La rencontre des deux personnages scelle la rencontre des âmes que Thor souhaitait, avec les complications qu'apporte la présence de la figure féminine d'Irène. Mais le sang n'est pas que le support d'un discours généalogique : les récurrences du terme dans Ægyptiaque, Fillette slovaque et Âmes blanches révèlent que Ritter met le sang en relation avec la violence physique, avec la sensualité et avec la religion. Violence et sensualité apparaissent étroitement liées, chez les hommes comme chez les femmes. Anička est ainsi confrontée à la sensualité romantique de Juro, à qui elle veut donner « sa chaleur, son sang, sa vie » (Ritter I903 : 343), et à l'assaut de Rudi, qui déclenche la rage : « tout son sang bouillonnait à l'idée d'avoir été léchée, mordue, étreinte par cet être à la nauséeuse pâleur » (70). Lors du viol que Neuenhaven fait subir à Ægyptiaque, le sang est le seul élément révélant la virginité de la jeune femme : «[il] ne s'était aperçu du viol qu'on le commettant » (Ritter I89I : 
I69). Dans Âmes blanches, c'est sur Raoul que s'abat la violence de la passion amoureuse : « il ne songea point à reprendre son mouchoir tout humide de son sang, et ne vit pas qu'Irène avait recueilli le lambeau sanglant, qu'elle l'avait furtivement baisé [...]. Ce sang de Raoul, dont elle séchait l'humidité sur son cœur» (Ritter I893: 154).

$\mathrm{Ce}$ « baiser du sang » rappelle des rituels religieux catholiques, ceux-là même qui ont fortement marqué Ritter pendant son enfance. En dernier lieu, c'est la symbolique du sang du Christ qui se dessine en filigrane derrière bien des pages de l'écrivain. Mais Ritter ne s'inscrit pas dans l'horizon moral que pourrait laisser supposer l'imaginaire catholique du sang, et il transgresse les normes du discours religieux canonique. C'est le cas par exemple lorsqu'il met Martin et Théodule sur le même plan que le Christ. Le premier de ces personnages affirme en effet : «Le sang de Jésus a coulé pour nous tous et un peu de mon sang a coulé pour Anička » (Ritter 1903: 345). La phrase a une portée d'autant plus blasphématoire que le sang de Martin est répandu sur un crucifix, et que celui-ci est assimilé à une relique à révérer : « il eut l'idée mystiquement voluptueuse de peindre tout son petit crucifix avec son sang "(288) pour ensuite l'offrir à Anička. Quant à Raoul, il suit le même parcours lorsque Théodule tombe malade : devant le crucifix, il se dit prêt à se sacrifier pour le salut du Christ qu'est à ses yeux son frêle camarade. Théodule devient ainsi l'emblème du Christ destiné à périr et que Raoul, son disciple, est appelé à sauver : « Il creva sans sourciller une des veines de son bras et écrivit avec son sang, un peu de son sang rouge trop épais, [...] avant de le rendre à l'anémié Théodule » (Ritter I893: I80).

Ce survol nous a permis de signaler combien la production de Ritter est traversée par le discours racial et par la hantise du sang, ce qui rend ses textes particulièrement intéressants pour qui voudrait les aborder sous un angle socio-ethnographique. En phase avec les théories de son temps, Ritter est surtout le vecteur d'une vision très positive des peuples slaves, qu'il évoque cependant de manière assez stéréotypée. Notre intention n'était ici que d'esquisser des pistes de recherche et de donner un aperçu d'une facette de l'œuvre encore peu explorée, en nous limitant du reste aux textes des débuts de l'écrivain. Une étude retraçant l'évolution du discours de Ritter sur la race et le sang tout au long de sa carrière ouvrirait certainement des perspectives insoupçonnées. 


\section{Bibliographie}

Donzé, Fernand, Calame, Caroline \& Charrière, Edmond, William Ritter au temps d'une autre Europe : dictionnaire à l'usage des curieux de la vie et de l'œuvre d'un Neuchâtelois hors du commun, Neuchâtel, Nouvelle Revue neuchâteloise (numéro monographique), 6I, I999.

Fournier-Facio, Gastón, Gustav Mahler. Il mio tempo verrà. La sua musica raccontata da critici, scrittori e interpreti. I9oI-20Io, Milano, Il Saggiatore, 20I0, pp. 3I7-333.

Galmiche, Xavier, « Europe et patries personnelles chez William Ritter », in Cultures d'Europe Centrale, $\mathrm{n}^{\circ}$ 3, Paris, 2003. En ligne : http://www.circe. paris-sorbonne.fr/spip.php?article56\&lang=fr (consulté le 23-IO-20I7)

—. «William et les garçons (d'Europe centrale) : "Aventures de volupté sous d'autres cieux" ", in Actes du colloque "L'Europe centrale en amateur. William Ritter (I867-I955) », 2I-22 novembre 2008, Paris, Centre interdisciplinaire de Recherches centre-européennes, Plateforme William Ritter, http://www.circe.paris-sorbonne.fr.

Gauthier, Cécile, «Malentendus autour de "l'âme slave” : plaidoyer pour une slavité centre-européenne », in Actes du colloque "L'Europe centrale en amateur. William Ritter (I867-I955) », 2I-22 novembre 2008, Paris, Centre interdisciplinaire de Recherches centre-européennes, Plateforme William Ritter, http://www.circe.paris-sorbonne.fr.

Listīkovā, Renáta, «William Ritter - homme de lettres et critique littéraire (ses grilles de lecture et ses critiques de la littérature tchèque) », in Actes du colloque «L'Europe centrale en amateur: William Ritter », 2I-22 novembre 2008.

Meylan, Claude, William Ritter, Chevalier de Gustav Mahler. Écrits, correspondance, documents, Peter Lang, 2000.

Riedlbauchovā, Tereza, «William Ritter et les écrivains tchèques : correspondance avec Jaroslav Vrchlický, Julius Zeyer et Svatopluk Čech (I894I906) ", in Actes du colloque « L'Europe centrale en amateur : William Ritter (I867-I955) », 2I-22 novembre 2008.

Ritter, William, Ægyptiaque, Neuchâtel, Nouvelle Revue neuchâteloise, 2000 [I89I].

-. Âmes blanches, Paris, Lemerre, I893.

-. Fillette slovaque, Paris, Mercure de France, 1903.

—. Boîte 39, Inv. II, I3-I6, « Pages tchèques », Ms, ALS, Berne.

—. Boîte 2, Inv., I.I, « La ballade des deux chemises sanglantes », Ms, ALS, Berne.

Ritter-Tcherv, Josef, «William Ritter peintre ", Ms, Bibliothèque de la Ville de La Chaux-de-Fonds, I953.

Szabō, Miloslav, « "The Victory of Swindle over the Folk's Beauty”. The Aesthetical Populism and Anti-Semitism of Ján Cádra », in Actes du colloque 
"L'Europe centrale en amateur. William Ritter (I867-I955) ", 2I-22 novembre 2008, Paris, Centre interdisciplinaire de Recherches centre-européennes, Plateforme William Ritter, http://www.circe.paris-sorbonne.fr. Taguieff, Pierre-André, La couleur et le sang : doctrines racistes à la française, Paris, Mille et une Nuit, 2002.

Toshiaki, Kozakaï \& Laurens, Stéphane, «Le mythe du "lien du sang" : une interprétation psychosociale de l'identité ethnique ", Nouvelle revue de psychologie 2006/2, pp. 187-200. 